Research Article

\title{
Solving the Multiobjective Transportation Decision-Making Problem Based on Improved S-Type Membership Function
}

\author{
Xiang Ziquan $(\mathbb{D}$, Yang Jiaqi, Muhammad Hamza Naseem $(\mathbb{D}$, and Xiang Zuquan \\ School of Transportation and Logistics Engineering, Wuhan University of Technology, Wuhan 430063, China \\ Correspondence should be addressed to Xiang Ziquan; 402053662@qq.com and Xiang Zuquan; xiangzuquan@whut.edu.cn
}

Received 14 July 2021; Accepted 18 August 2021; Published 9 September 2021

Academic Editor: Niansheng Tang

Copyright ( 2021 Xiang Ziquan et al. This is an open access article distributed under the Creative Commons Attribution License, which permits unrestricted use, distribution, and reproduction in any medium, provided the original work is properly cited.

\begin{abstract}
Aiming at the problem of multiobjective transportation decision-making, a fuzzy compromise method with an improved S-type membership function is proposed. This method not only considers a single objective and evaluates it marginally but also evaluates the overall objective as a whole. First of all, a multiobjective transportation decision-making model is established. Then, each objective function is mapped to a function on the interval $[0,1]$ through the S-type membership function, thereby transforming the multiobjective transportation linear programming model into a multiobjective transportation fuzzy compromise programming model, and its satisfaction is expressed by the global utility function. Finally, through two examples, the results of the example algorithm are compared with the results in the literature, highlighting the superiority of the method. The experimental results show that in the multiobjective transportation decision-making problem, the fuzzy compromise method of the S-type membership function has better flexibility and effectiveness.
\end{abstract}

\section{Introduction}

The single-objective linear programming decision-making problem is a traditional transportation problem. Many scholars have studied it and have formed a large number of effective solving algorithms. However, in actual transportation problems, multiobjective functions are generally considered such as the average delivery time of goods, the lowest cost, and the degree of damage to the product. The multiobjective transportation decision-making problem is not to simply pursue the optimization of a certain goal, but to comprehensively weigh the optimization problem of multiple goals. Zimmermann [1] first proposed the maximin method which was used to solve the fuzzy multiobjective linear programming problem. Current and Min [2, 3] reviewed and studied the multiobjective design of transportation networks. Climaco et al. [4] and Ringuest and Rinks [5] developed and studied interactive algorithms for multiobjective transportation decision-making problems. İlker Kolak et al. [6] studied the two-level multiobjective transportation optimization model with a sustainability perspective for intelligent transportation decision-making problems. Bai et al. [7] aimed at the decision-making problem of transportation and evaluated the vehicle performance and economic and environmental standards of the transportation fleet from the overall framework of sustainable development. Luo [8] used the multiobjective mixed integer programming method to study the multiobjective problem of transportation time, transportation cost, and transportation safety performance for the multiobjective problem of multimodal transportation route selection. Dong and Wan [9-11] studied the trapezoidal fuzzy multiobjective linear programming problem and its application in transportation problems, project portfolio selection, and other problems. Mateja š and Perić [12] proposed a new iterative method for multiobjective linear programming based on the principle of game theory. However, most of the above methods use linear membership functions to express the satisfaction of objective function. Although the calculation is simple, the solution is unstable, and the satisfaction of the global objective function is not evaluated. Based on this, this paper improves the logistics function (the image is an "S" shape) and proposes a fuzzy compromise programming method to improve the S-shaped membership function. At 
the same time, the global evaluation parameter is introduced to evaluate the global goal satisfaction. The S-shaped curve is chosen because although it is not as strict as the line type, it is flexible enough to fully describe the fuzzy parameters. The proposed fuzzy compromise programming method comprehensively contemplates the overall objective by marginal evaluation of a single objective and overall evaluation of all objectives. The examples show the effectiveness and convergence of method. The contributions of this paper are as follows:

(1) This paper improves the "logistic function," introduces it into a multiobjective transportation decision-making model, and uses it to express membership function.

(2) In the past, most of the multiobjective functions only considered the importance of a single-objective function and did not consider the inherent correlation between the objective functions. However, this paper uses the global utility function of improved S-type membership degree which is used to represent the total satisfaction degree of decision-makers, which not only considers the evaluation of a single objective but also considers the internal correlation between the objective functions. Therefore, it has better application prospects.

The construction of this paper is as follows: Section 2 introduces the multiobjective transportation decisionmaking problem and briefly introduces nondominated solutions and optimal compromise solutions. Section 3 proposes a fuzzy compromise programming method based on an improved S-type membership function. Section 4 gives two examples in reference. Section 5 gives the conclusion of this paper.

\section{Multiobjective Transportation Decision- Making Problem}

2.1. Model Establishment. The multiobjective transportation decision-making problem with $m$ starting points and $n$ ending point is considered. Assume that the total supply at the starting point is equal to the total demand at the destination. Now, it is necessary to transport the same product from each starting point to each destination, and at the same time, make the corresponding penalty cost (transportation cost, delivery time, delivered transportation quantity, product damage degree, etc.) lowest. Define the following description symbols:

(i) $i$ indicates the starting point $i$ indicator, namely, $i \in\{1,2, \ldots, m\}$.

(ii) $j$ indicates the endpoint $j$ indicator, namely, $j \in\{1,2, \ldots, n\}$.

(iii) $q$ represents the objective function $q$ indicator, namely, $q \in\{1,2, \ldots, Q\}$.

(iv) $c_{i j}^{q}$ indicates the unit penalty cost of the $q$-th objective function, starting point $i$ transport to the destination $j$. (v) $x_{i j}$ indicates the number of products from the starting point $i$ transport to the destination $j$.

(vi) $a_{i}$ indicates the total number of products supplied from starting point $i$.

(vii) $b_{i}$ indicates the total number of products required at the destination $j$.

According to the above-agreed symbols, the multiobjective transportation decision-making problem can be modeled as follows:

$$
\begin{aligned}
& \operatorname{Min} f_{q}=\sum_{i=1}^{m} \sum_{i=1}^{n} c_{i j}^{q} x_{i j}, \quad c_{i j}^{q} \geq 0, q=1, \ldots, Q, \\
& \text { s.t. }\left\{\begin{array}{l}
\sum_{i=1}^{m} x_{i j}=b_{i}, \quad \forall j \in\{1, \ldots, n\}, \\
\sum_{j=1}^{n} x_{i j}=a_{i}, \quad \forall i \in\{1, \ldots, m\}, \\
\sum_{i=1}^{m} a_{i}=\sum_{j=1}^{n} b_{j}, \\
x_{i j} \geq 0 .
\end{array}\right.
\end{aligned}
$$

In the multiobjective transportation models (1) and (2), (1) represents the $Q$ objective functions and (2) represents constraint condition. The model has $m \times n$ variables, and $m+n$ constraint equations (1) and (2) are single-objective balanced transportation decision-making problems when $q=1$.

2.2. Nondominated Solution Set. In (1) and (2), if there is a feasible solution $x_{i j}^{*} \in X$, then all other feasible solutions are $x_{i j} \in X$, and it conforms to the following inequality:

$$
\sum_{i=}^{m} \sum_{j=}^{n} c_{i j}^{q} x_{i j}^{*} \leq \sum_{i=}^{m} \sum_{j=}^{n} c_{i j}^{q} x_{i j}, \quad q \in\{1,2, \ldots, Q\} .
$$

At the same time, at least one inequality is strictly established, namely,

$$
\sum_{i=1}^{m} \sum_{j=1}^{n} c_{i j}^{q} x_{i j}^{*}<\sum_{i=1}^{m} \sum_{j=1}^{n} c_{i j}^{q} x_{i j}
$$

Then, $x_{i j}^{*}$ is called the nondominated solution, and the set of all nondominated solutions is called the nondominated solution set.

2.3. Optimal Compromise Solution. In most cases, due to conflicts among various objective functions, decisionmakers choose to assign different weights to each different objective according to their actual preferences to obtain the optimal solution. According to the definition of a nondominated solution, it is generally accepted that the optimal compromise solution must be a nondominated solution which is the optimal solution in a sense. 


\section{Multiobjective Transportation Decision Model Solution}

The above model contains multiobjective functions, and the unit of the dimension of the objective function is sometimes inconsistent. It is not advisable to simply use linear weighting to obtain the value, and at the same time, it cannot achieve all the optimal values of objective functions (the objective functions of this paper are the minimum value), which is usually not achieved. This paper attempts to find an optimal compromise solution. In this optimal compromise solution, the global evaluation of the comprehensive membership degree of all objectives is the largest, which means that global satisfaction is the largest. Therefore, to solve this model, a fuzzy compromise programming method with an improved S-type membership function is proposed, and the multiobjective linear programming model is transformed into a multiobjective nonlinear fuzzy compromise programming model. In this model, the global utility function of the S-type membership is used to represent the global satisfaction of decision-makers. This paper uses S-curve because it is not as strict as linear form, but it has strong flexibility and high robustness, and can fully describe the fuzzy parameter [13-15].

3.1. Improved S-Type Membership Function. Create a mapping, there is $\varphi_{x}: X \longrightarrow[0,1]$, and among them, $x \in[L, U](L<U), \varphi_{x} \in[0,1]$. For any value of $x$, it has a unique number in the interval $[0,1] . X \longrightarrow[0,1]$ determines a fuzzy subset $\varphi_{x}$ of $X$, and $\varphi_{x}$ is called the membership function of $x$. The target decision rule is to select the solution with the highest membership in the decision set. In this paper, the improved logistic function is used as the membership function $[16,17]$. The image of the improved logistic function is like an "S" shape, so it is called the improved S-type membership function. Its membership function is shown in (3) which is as follows:where $\varphi_{x}$ is the S-type membership function, $L$ represents the minimum value of $x$, and $U$ represents the maximum value of $x . K$ is a positive number which determines the shape of the membership function, and the larger the value of $K$, the smaller the fuzziness is. The membership function values are defined as $\varphi \in[0.001,0.999]$, and the value of $x$ changes in the interval $[L, U]$. This value interval is selected because the probability of taking $L$ and $M$ values is very low in the multiobjective transportation decision-making problem.

$$
\varphi_{x}= \begin{cases}1, & x<L, \\ 0.999, & x=L, \\ \frac{B}{1+C e^{K[(x-L) /(U-L)]},} & x \in(L, U), \\ 0.001, & x=U, \\ 0, & x>U,\end{cases}
$$

According to the definition of function limit, if $x \longrightarrow L$, then

$$
B=0.999(1+C)
$$

If $x \longrightarrow U$, then

$$
B=0.001\left(1+C e^{K}\right) .
$$

When $x=(L+U) / 2$ and $\varphi(x)=0.5$ (this value is determined experimentally according to the actual situation, and 0.5 is taken in this paper), then

$$
B=0.5\left(1+C e^{0.5 K}\right)
$$

Simultaneous equations (6)-(8) can be obtained:

$$
B=1, C=0.001, \quad \text { with } K=13.813 \text {. }
$$

Therefore, the improved S-type membership function image is shown in Figure 1.

3.2. S-Type Membership Fuzzy Compromise Programming Method. In (1), for a single-objective function $f_{q}(q=1, \ldots, Q)$, the optimal value is obtained which is denoted as $L_{q}$. If $x_{q}^{*}$ is the solution of a single-objective function $f_{q}(x)$, so

$$
L_{q}=f_{q}\left(x_{q}^{*}\right)=\min f_{q}(x), \quad q \in\{1,2, \ldots, Q\} .
$$

Each objective function $f_{q}$ can form a pay-off matrix with $Q$ rows and $Q$ columns under $x_{q}^{*}$, and the payoff matrix can be expressed as follows: Table 1

Through the pay-off matrix, the maximum value $U_{q}$ and minimum value $L_{q}$ of each objective function $f_{q}$ can be obtained:

$$
\begin{aligned}
L_{q}= & f_{q}\left(x_{q}^{*}\right), \quad U_{q}=\max \left[f_{q}\left(x_{1}^{*}\right), f_{q}\left(x_{2}^{*}\right), \ldots, f_{q}\left(x_{Q}^{*}\right)\right] \\
& \cdot(q=1, \ldots, Q) .
\end{aligned}
$$

The S-type membership function is used to express the membership degree of the objective function $f_{q}(q=1, \ldots, Q)$, namely,

$$
\phi_{f_{1}}= \begin{cases}1, & f_{1}<L_{1}, \\ 0.999, & f_{1}=L_{1}, \\ \frac{1}{1+10^{-3} \times e^{13.813 \times\left[\left(f_{1}-L_{1}\right) /\left(U_{1}-L_{1}\right)\right]},} & f_{1} \in\left(L_{1}, U_{1}\right), \\ 0.001, & f_{1}=U_{1}, \\ 0, & f_{1}>U_{1},\end{cases}
$$




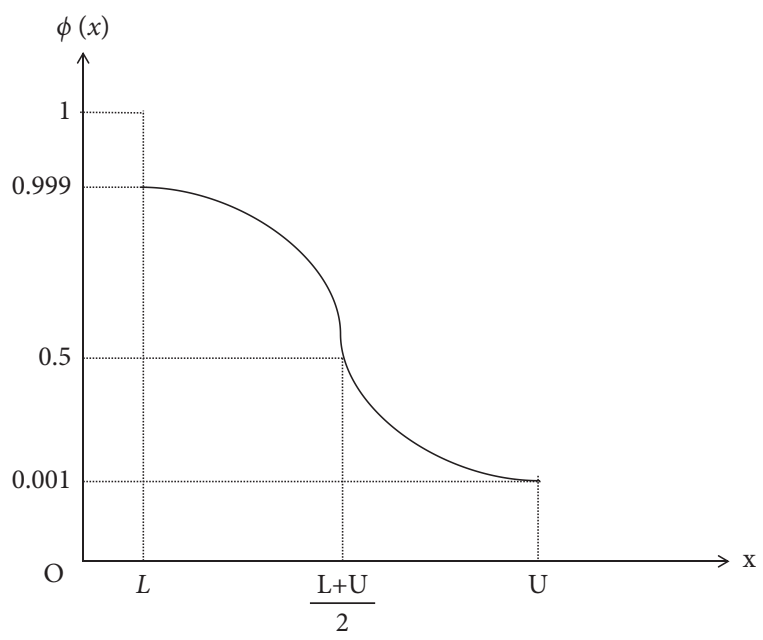

Figure 1: S-type membership function image.

TABLE 1: Pay-off matrix of objective functions.

\begin{tabular}{ccccc}
\hline & $x_{1}^{*}$ & $x_{2}^{*}$ & $\ldots$ & $x_{Q}^{*}$ \\
\hline$f_{1}$ & $f_{1}\left(x_{1}^{*}\right)$ & $f_{1}\left(x_{2}^{*}\right)$ & $\ldots$ & $f_{1}\left(x_{Q}^{*}\right)$ \\
$f_{2}$ & $f_{2}\left(x_{1}^{*}\right)$ & $f_{2}\left(x_{2}^{*}\right)$ & $\ldots$ & $f_{2}\left(x_{Q}^{*}\right)$ \\
$\ldots$ & $\ldots$ & $\ldots$ & $\ldots$ & $\ldots$ \\
$f_{Q}$ & $f_{Q}\left(x_{1}^{*}\right)$ & $f_{Q}\left(x_{2}^{*}\right)$ & $\ldots$ & $f_{Q}\left(x_{Q}^{*}\right)$ \\
\hline
\end{tabular}

$\phi_{f_{2}}= \begin{cases}1, & f_{2}<L_{2}, \\ 0.999, & f_{2}=L_{2}, \\ \frac{1}{1+10^{-3} \times e^{13.813 \times\left[\left(f_{2}-L_{2}\right) /\left(U_{2}-L_{2}\right)\right]},} & f_{2} \in\left(L_{2}, U_{2}\right), \\ 0.001, & f_{2}=U_{2}, \\ 0, & f_{2}>U_{2},\end{cases}$

$$
\phi_{f_{Q}}= \begin{cases}1, & f_{Q}<L_{Q}, \\ 0.999, & f_{Q}=L_{Q}, \\ 1+10^{-3} \times e^{13.813 \times\left[\left(f_{Q}-L_{Q}\right) /\left(U_{Q}-L_{Q}\right)\right]}, & f_{Q} \in\left(L_{Q}, U_{Q}\right), \\ 0.001, & f_{Q}=U_{Q}, \\ 0, & f_{Q}>U_{Q} .\end{cases}
$$

The decision-makers evaluate the importance of each objective function and assign a weight $w_{q}$ to each objective function $f_{q}$ and meet the normalization condition, and then we have

$$
w=\left(w_{1}, \ldots, w_{q}, \ldots, w_{Q}\right), \quad w_{q} \geq 0 \text {, and } \sum_{q=1}^{Q} w_{q}=1
$$

According to the weight proportion and $\Phi_{f_{q}}$, a multiobjective transportation fuzzy compromise programming model with an improved S-type membership function is established:

$$
\begin{aligned}
& M_{w}^{\alpha}\left(\phi_{f_{1}}, \ldots, \phi_{f_{Q}}\right)= M a x\left(\sum_{q=1}^{Q} w_{q} \phi_{f_{q}}^{\alpha}\right)^{1 / \alpha} \quad(0<|\alpha|<\infty), \\
& \operatorname{s.t}\left\{\begin{array}{l}
\sum_{i=1}^{m} x_{i j}=b_{i}, \quad \forall j \in\{1, \ldots, n\}, \\
\sum_{j=1}^{n} x_{i j}=a_{i}, \quad \forall i \in\{1, \ldots, m\}, \\
\sum_{i=1}^{m} a_{i}=\sum_{j=1}^{n} b_{j}, \\
x_{i j} \geq 0 .
\end{array}\right.
\end{aligned}
$$

(14) represents the global utility function, which is the satisfaction degree of the overall objective. $\alpha$ represents the global evaluation parameter. Generally, the value of $\alpha$ was taken as 1 in the literature, and although the feasible solution of each objective function and the importance of each objective function are considered, the internal correlation between each objective function is not considered [18-20]. (14) considers not only the satisfaction degree of a single objective but also the internal correlation between each objective. In other words, considering the multiobjective optimization problem from a global perspective, it has greater practical value. Here are some forms of global utility functions, where

(1) when $\alpha \longrightarrow 0, M_{w}^{0}\left(\phi_{f_{1}}, \ldots, \phi_{f_{Q}}\right)=\prod_{q=1}^{Q}\left(\phi_{f_{q}}\right)^{w_{q}}$,

(2) when $\alpha=1, M_{w}^{1}\left(\varphi_{f_{1}}, \ldots, \phi_{f_{Q}}\right)=\sum_{q=1}^{Q} w_{q} \phi_{f_{q}}$,

(3) when $\alpha=2, M_{w}^{2}\left(\phi_{f_{1}}, \ldots, \phi_{f_{Q}}\right)=\left(\sum_{q=1}^{Q} w_{q} \phi_{f_{q}}\right)^{1 / 2}$,

(4) when $\alpha \longrightarrow+\infty$ and $w_{1}=w_{2}=\cdots=w_{Q}=1 / Q$, $M_{w}^{+\infty}\left(\phi_{f_{1}}, \ldots, \phi_{f_{Q}}\right)=\operatorname{Max}_{1 \leq q \leq Q}\left\{\phi_{f_{q}}\right\}$, and

(5) when $\alpha \longrightarrow-\infty$ and $w_{1}=w_{2}=\cdots=w_{Q}=1 / Q$, $M_{w}^{-\infty}\left(\phi_{f_{1}}, \ldots, \phi_{f_{Q}}\right)=\operatorname{Min}_{1 \leq q \leq Q}\left\{\phi_{f_{q}}\right\}$.

$M_{w}^{-\infty}\left(\phi_{f_{1}}, \ldots, \phi_{f_{Q}}\right)$ is often used in multiobjective decision-making problems in engineering field [21-23], so it is mainly discussed below. Let $\lambda_{q}=\varphi_{f_{q}} \lambda=\min _{1 \leq q \leq Q}\left\{\lambda_{q}\right\}$, $q \in\{1,2, \ldots, Q\}$. In this case, the fuzzy compromise programming model of multiobjective transportation, i.e., (14), is equivalent to the following model: 
$\operatorname{Max} \lambda$,

\section{Among them,}

$$
\text { s.t. }\left\{\begin{array}{l}
\lambda \leq \frac{1}{1+10^{-3} \times e^{13.813 \times\left[\left(f_{q}-L_{q}\right) /\left(U_{q}-L_{q}\right)\right]}, \quad q \in\{1,2, \ldots, Q\}} \\
\sum_{i=1}^{m} x_{i j}=b_{i}, \quad \forall j \in\{1, \ldots, n\}, \\
\sum_{j=1}^{n} x_{i j}=a_{i}, \quad \forall i \in\{1, \ldots, m\}, \\
\sum_{i=1}^{m} a_{i}=\sum_{j=1}^{n} b_{j}, \\
x_{i j} \geq 0 .
\end{array}\right.
$$

$$
\begin{aligned}
& \lambda \leq \frac{1}{1+10^{-3} \times e^{13.813 \times\left[\left(f_{q}-L_{q}\right) /\left(U_{q}-L_{q}\right)\right]}} \\
& \Rightarrow \lambda+10^{-3} \times \lambda e^{13.813 \times\left[\left(f_{q}-L_{q}\right) /\left(U_{q}-L_{q}\right)\right]} \leq 1, \\
& \Rightarrow 10^{-3} \times \lambda e^{13.813 \times\left[\left(f_{q}-L_{q}\right) /\left(U_{q}-L_{q}\right)\right]} \leq 1-\lambda \\
& \Rightarrow e^{13.813 \times\left[\left(f_{q}-L_{q}\right) /\left(U_{q}-L_{q}\right)\right]} \leq \frac{(1-\lambda)}{\left(10^{-3} \times \lambda\right)} \\
& \Rightarrow 13.813 \times\left[\frac{\left(f_{q}-L_{q}\right)}{\left(U_{q}-L_{q}\right)}\right] \leq \operatorname{In} \frac{(1-\lambda)}{\left(10^{-3} \times \lambda\right)} \\
& \Rightarrow 13.813 \times\left[\frac{\left(f_{q}-L_{q}\right)}{\left(U_{q}-L_{q}\right)}\right] \leq \operatorname{In} \frac{(1-\lambda)}{\lambda}-\operatorname{In} 10^{-3} \\
& \Rightarrow 13.813 \times\left[\frac{\left(f_{q}-L_{q}\right)}{\left(U_{q}-L_{q}\right)}\right]+\operatorname{In} 10^{-3} \leq \operatorname{In} \frac{(1-\lambda)}{\lambda} \text {. }
\end{aligned}
$$

Let $\eta=\operatorname{In}(1-\lambda) / \lambda$, then (15) can be simplified as 
$\operatorname{Min} \eta$,

$$
\operatorname{s.t}\left\{\begin{array}{l}
\eta \geq 13.813 \times\left[\frac{\left(f_{q}-L_{q}\right)}{\left(U_{q}-L_{q}\right)}\right]+I n 10^{-3}, \quad q \in\{1,2, \ldots, Q\}, \\
\sum_{i=1}^{m} x_{i j}=b_{i}, \quad \forall j \in\{1, \ldots, n\}, \\
\sum_{j=1}^{n} x_{i j}=a_{i}, \quad \forall i \in\{1, \ldots, m\}, \\
\sum_{i=1}^{m} a_{i}=\sum_{j=1}^{n} b_{j}, \\
x_{i j} \geq 0 .
\end{array}\right.
$$

\subsection{The Algorithm.}

(i) Step 1: for a single-objective function $f_{q}(q=1, \ldots, Q)$, the optimal value $L_{q}$ and solution $x_{q}^{*}$ of the objective function are calculated.

(ii) Step 2: according to the optimal solution $\left(x_{q}^{*}\right)$ of the single-objective function in Step 1, the pay-off matrix is obtained.

(iii) Step 3: according to the pay-off matrix in Step 2, the maximum value $U_{q}$ and minimum value $L_{q} \quad(q=$ $1, \ldots, Q)$ of each objective function are determined.

(iii) Step 4: according to $U_{q}$ and $L_{q}$ of Step 3, the S-type membership function corresponding to each objective function $\varphi_{f_{q}}(q=1, \ldots, Q)$ is determined.

(iv) Step 5: according to the preference of decisionmakers, the weight proportion value $\omega$ and the global utility factor $\alpha$ are reasonably determined. The optimal solution $x^{*}$ is determined by using MATLAB software or an intelligent optimization algorithm, and $x^{*}$ is the optimal compromise solution of the original multiobjective transportation decision-making problem.

\section{Case Analysis}

Example 1. In order to verify the effectiveness of the method, the multiobjective transportation decision model in reference $[21,22]$ is considered:

$$
\begin{aligned}
& \text { Min } f_{1}=16 x_{11}+19 x_{12}+12 x_{13}+22 x_{21}+13 x_{22}+19 x_{23}+14 x_{31}+28 x_{32}+8 x_{33}, \\
& \text { Min } f_{2}=9 x_{11}+14 x_{12}+12 x_{13}+16 x_{21}+10 x_{22}+14 x_{23}+8 x_{31}+20 x_{32}+6 x_{33}, \\
& \text { s.t. }\left\{\begin{array}{l}
\sum_{j=1}^{3} x_{1 j}=14, \sum_{j=1}^{3} x_{2 j}=16, \sum_{j=1}^{3} x_{3 j}=12, \\
\sum_{i=1}^{3} x_{i 1}=10, \sum_{i=1}^{3} x_{i 2}=15, \sum_{i=1}^{3} x_{i 3}=17, \\
x_{i j} \geq 0, \quad i=1,2,3 ; j=1,2,3 .
\end{array}\right.
\end{aligned}
$$

(i) Step 1: for the objective function $f_{1}, L_{1}=517$, $x_{1}^{\bullet}=\left\{\begin{array}{l}x_{11}=9, x_{13}=5, x_{21}=1, x_{22}=15, x_{33}=12, \\ x_{12}=x_{23}=x_{31}=x_{32}=0 .\end{array}\right.$

(ii) For the objective function $f_{2}, L_{2}=374$,

$$
x_{2}^{\bullet}=\left\{\begin{array}{l}
x_{11}=10, x_{13}=4, x_{22}=15, x_{23}=1, x_{33}=12, \\
x_{12}=x_{21}=x_{31}=x_{32}=0 .
\end{array}\right.
$$

(iii) Step 2: According to Table 1 and step 1 in Example 1 , the pay-off matrix of $\mathrm{f} 1$ and $\mathrm{f} 2$ can be obtained, and the results are shown in Table 2. (iv) Step 3: according to the pay-off matrix in Step 2, get the maximum and minimum values:

$$
\begin{aligned}
& L_{1}=517, \\
& U_{1}=518 ; \\
& L_{2}=374, \\
& U_{2}=379 .
\end{aligned}
$$

(v) Step 4: according to the results of Step 3, the S-type membership function expressions of the two objective functions are as follows: 
TABle 2: Pay-off matrix of $f_{1}$ and $f_{2}$ of Example 1.

\begin{tabular}{lcc}
\hline & $x_{1}^{*}$ & $x_{2}^{*}$ \\
\hline$f_{1}$ & 517 & 518 \\
$f_{2}$ & 379 & 374 \\
\hline
\end{tabular}

$$
\begin{aligned}
& \begin{cases}1, & f_{1}<517 \\
0.999, & f_{1}=517\end{cases} \\
& \varphi_{f_{1}}= \begin{cases}\frac{1}{1+10^{-3} \times e^{13.813 \times\left[\left(f_{1}-L_{1}\right) /\left(U_{1}-L_{1}\right)\right]},} & f_{1} \in(517,518), \\
0.001, & f_{1}=518,\end{cases} \\
& f_{1}>518 \text {, } \\
& \varphi_{f_{2}}= \begin{cases}1, & f_{2}<374, \\
0.999, & f_{2}=374, \\
\frac{1}{1+10^{-3} \times e^{13.813 \times\left[\left(f_{2}-L_{2}\right) /\left(U_{2}-L_{2}\right)\right]},} & f_{2} \in(374,379), \\
0.001, & f_{2}=379, \\
0, & f_{2}>379 .\end{cases}
\end{aligned}
$$

(vi) Step 5: in order to compare with the calculation results of reference [21, 22], the global evaluation parameter of this example is $\alpha \longrightarrow-\infty$ and the weight vector is $w_{1}=w_{2}=1 / 2$, and then according to (15) and (17), (18) is transformed into the following fuzzy compromise programming model:

Min $\eta$,

$$
s . t\left\{\begin{array}{l}
\eta \geq 13.813 \times\left[\frac{\left(f_{q}-L_{q}\right)}{\left(U_{q}-L_{q}\right)}\right]+\operatorname{In} 10^{-3}, \quad q=1,2, \\
\sum_{j=1}^{3} x_{1 j}=14, \sum_{j=1}^{3} x_{2 j}=16, \sum_{j=1}^{3} x_{3 j}=12, \\
\sum_{i=1}^{3} x_{i 1}=10, \sum_{i=1}^{3} x_{i 2}=15, \sum_{i=1}^{3} x_{i 3}=17, \\
x_{i j} \geq 0, \quad i=1,2,3 ; j=1,2,3,
\end{array}\right.
$$

(vii) where $\lambda_{q}=\varphi_{f_{q}}(q=1,2), \quad \lambda=\min _{1 \leq q \leq 2}\left\{\lambda_{q}\right\}$, and $\eta=\operatorname{In}(1-\lambda) / \lambda$. The optimal value $\left(f_{1}, f_{2}\right)=(517.5,376.5)$ is obtained by MATLAB software:

$$
x^{\bullet}=\left\{\begin{array}{l}
x_{11}=9.5, x_{13}=4, x_{21}=0.5, x_{22}=15, x_{23}=0.5, x_{33}=12, \\
x_{12}=x_{31}=x_{32}=0 .
\end{array}\right.
$$

(viii) $\lambda=0.5$, and $\eta=0$. The optimal value is completely consistent with the results of the literature [21, 22], which shows the effectiveness of the improved S-type membership function method.

Example 2. To verify the effectiveness of the method, consider the multiobjective transportation decision model in reference [23]:

$$
\begin{aligned}
& \text { Min } f_{1}=x_{11}+2 x_{12}+7 x_{13}+7 x_{14}+x_{21}+9 x_{22}+3 x_{23}+4 x_{24}+8 x_{31}+9 x_{32}+4 x_{33}+6 x_{34}, \\
& \text { Min } f_{2}=4 x_{11}+4 x_{12}+3 x_{13}+3 x_{14}+5 x_{21}+8 x_{22}+9 x_{23}+10 x_{24}+6 x_{31}+2 x_{32}+5 x_{33}+x_{34}, \\
& \text { s.t. }\left\{\begin{array}{l}
\sum_{j=1}^{4} x_{1 j}=8, \sum_{j=1}^{4} x_{2 j}=19, \sum_{j=1}^{4} x_{3 j}=17, \\
\sum_{i=1}^{3} x_{i 1}=11, \sum_{i=1}^{3} x_{i 2}=3, \sum_{i=1}^{3} x_{i 3}=14, \sum_{i=1}^{3} x_{i 4}=16, \\
x_{i j} \geq 0, \quad i=1,2,3 ; j=1,2,3,4 .
\end{array}\right.
\end{aligned}
$$

(i) Step 1: for the objective function $f_{1}, L_{1}=143$,

$$
x_{1}^{\bullet}=\left\{\begin{array}{l}
x_{11}=5, x_{12}=3, x_{21}=6, \\
x_{24}=13, x_{33}=14, x_{34}=3, \\
x_{22}=x_{32}=x_{31}=x_{13}=x_{23}=x_{14}=0 .
\end{array}\right.
$$

(ii) For the objective function $f_{2}, L_{2}=167$,

$$
x_{2}^{\bullet}=\left\{\begin{array}{l}
x_{13}=8, x_{21}=11, x_{22}=2, \\
x_{23}=6, x_{32}=1, x_{34}=16, \\
x_{11}=x_{12}=x_{31}=x_{33}=x_{14}=x_{24}=0 .
\end{array}\right.
$$


(iii) Step 2: According to Table 1 and step 1 in Example 2 , the pay-off matrix of $\mathrm{f} 1$ and $\mathrm{f} 2$ can be obtained, and the results are shown in Table 3.

(iv) Step 3: according to the pay-off matrix in Step 2, the maximum and minimum values are as follows:

$$
\begin{gathered}
L_{1}=143, \\
U_{1}=208 ; \\
L_{2}=167, \\
U_{2}=265 .
\end{gathered}
$$

(v) Step 4: according to the results of Step 3, the S-type membership function expressions of the two objective functions are as follows:

$$
\begin{aligned}
& \begin{cases}1, & f_{1}<143, \\
0.999, & f_{1}=143,\end{cases}
\end{aligned}
$$

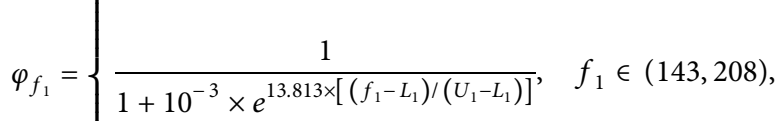

$$
\begin{aligned}
& \text { 0.001, } \quad f_{1}=208 \text {, } \\
& 0, \quad f_{1}>208, \\
& \varphi_{f_{2}}= \begin{cases}1, & f_{2}<167, \\
0.999 & f_{2}=167, \\
\frac{1}{1+10^{-3} \times e^{13.813 \times\left[\left(f_{2}-L_{2}\right) /\left(U_{2}-L_{2}\right)\right]},} & f_{2} \in(167,265), \\
0.001, & f_{2}=265, \\
0, & f_{2}>265 .\end{cases}
\end{aligned}
$$

According to the weight ratio $\left(w_{1}, w_{2}\right)$ and global evaluation parameter $\alpha$, the multiobjective transportation decision-making model can be changed into the following fuzzy compromise programming model:

$$
\begin{aligned}
& M_{w}^{\alpha}\left(\varphi_{f_{1}}, \varphi_{f_{2}}\right)= \operatorname{Max}\left(w_{1} \Phi_{f_{1}}^{\alpha}+w_{2} \Phi_{f_{2}}^{\alpha}\right)^{1 / \alpha}, \\
& \text { s.t. }\left\{\begin{array}{l}
\sum_{j=1}^{4} x_{1 j}=8, \sum_{j=1}^{4} x_{2 j}=19, \sum_{j=1}^{4} x_{3 j}=17, \\
\sum_{i=1}^{3} x_{i 1}=11, \sum_{i=1}^{3} x_{i 2}=3, \sum_{i=1}^{3} x_{i 3}=14, \sum_{i=1}^{3} x_{i 4}=16, \\
x_{i j} \geq 0, \quad i=1,2,3 ; j=1,2,3,4 .
\end{array}\right.
\end{aligned}
$$

In this paper, MATLAB software is used to solve the problem, and the calculation results of the model are given under different weights and global evaluation parameter $\alpha$ value. Considering the actual situation, the
TABle 3: Pay-off matrix of $f_{1}$ and $f_{2}$ of Example 2.

\begin{tabular}{lcc}
\hline & $x_{1}^{*}$ & $x_{2}^{*}$ \\
\hline$f_{1}$ & 143 & 208 \\
$f_{2}$ & 265 & 167 \\
\hline
\end{tabular}

TABle 4: The optimal compromise solution for different weight ratios $(\alpha=0)$.

\begin{tabular}{lcccc}
\hline & $(0.0,1.0)$ & $(0.05,0.95)$ & $(0.1,0.9)$ & $(0.2,0.8)$ \\
\hline$f_{1}$ & 208 & 168 & 168 & 164 \\
$f_{2}$ & 167 & 185 & 185 & 190 \\
$\Phi_{f_{1}}$ & 0.001 & 0.8312 & 0.8312 & 0.9201 \\
$\Phi_{f_{2}}$ & 0.9875 & 0.9750 & 0.9750 & 0.9507 \\
\hline & $(0.3,0.7)$ & $(0.4,0.6)$ & $(0.5,0.5)$ & $(0.6,0.4)$ \\
$f_{1}$ & 164 & 160 & 160 & 160 \\
$f_{2}$ & 190 & 195 & 195 & 195 \\
$\Phi_{f_{1}}$ & 0.9201 & 0.9642 & 0.9642 & 0.9642 \\
$\Phi_{f_{2}}$ & 0.9750 & 0.9507 & 0.9507 & 0.9507 \\
\hline & $(0.7,0.3)$ & $(0.8,0.2)$ & $(0.9,0.1)$ & $(1.0,0.0)$ \\
$f_{1}$ & 160 & 156 & 156 & 143 \\
$f_{2}$ & 195 & 200 & 200 & 265 \\
$\Phi_{f_{1}}$ & 0.9642 & 0.9844 & 0.9844 & 0.999 \\
$\Phi_{f_{2}}$ & 0.9507 & 0.9051 & 0.9051 & 0.001 \\
\hline
\end{tabular}

TABLE 5: The optimal compromise solution for different weight ratios $(\alpha=1)$.

\begin{tabular}{lcccc}
\hline & $(0.0,1.0)$ & $(0.05,0.95)$ & $(0.1,0.9)$ & $(0.2,0.8)$ \\
\hline$f_{1}$ & 208 & 176 & 168 & 164 \\
$f_{2}$ & 167 & 175 & 185 & 190 \\
$\Phi_{f_{1}}$ & 0.001 & 0.4735 & 0.8312 & 0.9201 \\
$\Phi_{f_{2}}$ & 0.999 & 0.9969 & 0.9875 & 0.9750 \\
\hline & $(0.3,0.7)$ & $(0.4,0.6)$ & $(0.5,0.5)$ & $(0.6,0.4)$ \\
$f_{1}$ & 164 & 160 & 160 & 156 \\
$f_{2}$ & 190 & 195 & 195 & 200 \\
$\Phi_{f_{1}}$ & 0.9201 & 0.9642 & 0.9642 & 0.9844 \\
$\Phi_{f_{2}}$ & 0.9750 & 0.9507 & 0.9507 & 0.9051 \\
\hline & $(0.7,0.3)$ & $(0.8,0.2)$ & $(0.9,0.1)$ & $(1.0,0.0)$ \\
$f_{1}$ & 156 & 156 & 156 & 143 \\
$f_{2}$ & 200 & 200 & 200 & 265 \\
$\Phi_{f_{1}}$ & 0.9844 & 0.9844 & 0.9844 & 0.999 \\
$\Phi_{f_{2}}$ & 0.9051 & 0.9051 & 0.9051 & 0.001 \\
\hline & & & &
\end{tabular}

literature [23] takes $x_{\mathrm{ij}}$ as a nonnegative integer, so this example only considers integer solutions. Table 4 shows the optimal compromise solution under different weights when $\alpha=0$. Table 5 shows the optimal compromise solution under different weights when $\alpha=1$. Table 6 shows the optimal compromise solution under different weights when $\alpha=2$. When $\alpha \longrightarrow-\infty, f_{1}=160$, $f_{2}=195, \phi_{f_{1}}=0.9642, \phi_{f_{2}}=0.9507$. The optimal compromise solution in this example depends on the preference of the decision-makers, as can be seen from Tables 4-6:

(1) When the decision-maker considers that the satisfaction of $f_{1}$ and $f_{2}$ is almost the same, that is, they 
TABLE 6: The optimal compromise solution for different weight ratios $(\alpha=2)$.

\begin{tabular}{lcccc}
\hline & $(0.0,1.0)$ & $(0.05,0.95)$ & $(0.1,0.9)$ & $(0.2,0.8)$ \\
\hline$f_{1}$ & 208 & 172 & 168 & 164 \\
$f_{2}$ & 167 & 180 & 185 & 190 \\
$\Phi_{f_{1}}$ & 0.001 & 0.6779 & 0.8312 & 0.9201 \\
$\Phi_{f_{2}}$ & 0.999 & 0.9938 & 0.9875 & 0.9750 \\
\hline & $(0.3,0.7)$ & $(0.4,0.6)$ & $(0.5,0.5)$ & $(0.6,0.4)$ \\
$f_{1}$ & 164 & 160 & 160 & 160 \\
$f_{2}$ & 190 & 195 & 195 & 195 \\
$\Phi_{f_{1}}$ & 0.9201 & 0.9642 & 0.9642 & 0.9642 \\
$\Phi_{f_{2}}$ & 0.9750 & 0.9507 & 0.9507 & 0.9507 \\
\hline & $(0.7,0.3)$ & $(0.8,0.2)$ & $(0.9,0.1)$ & $(1.0,0.0)$ \\
$f_{1}$ & 156 & 156 & 156 & 143 \\
$f_{2}$ & 200 & 200 & 200 & 265 \\
$\Phi_{f_{1}}$ & 0.9844 & 0.9844 & 0.9844 & 0.999 \\
$\Phi_{f_{2}}$ & 0.9051 & 0.9051 & 0.9051 & 0.001 \\
\hline
\end{tabular}

are equally important, then $(160,195)$ is the optimal compromise solution. Currently, the corresponding satisfaction degree is $(0.9642,0.9507)$, and the satisfaction degree exceeds 0.95 .

(2) When the decision-maker considers that the satisfaction $f_{1}$ is more than $f_{2}$, the optimal compromise solution is $(156,200)$. At this time, the corresponding satisfaction degree is $(0.9844,0.9051)$, and the satisfaction is more than 0.9 . The satisfaction of $f_{1}$ is 0.9844, which indicates that the decision-makers prefer to $f_{1}$.

(3) When the decision-maker considers that satisfaction $f_{2}$ exceeds $f_{1}$, the optimal compromise solution is $(164,190)$, and the corresponding satisfaction degree is $(0.9201,0.9750)$, which is more than 0.9 , and the satisfaction degree $f_{2}$ is 0.9750 , which indicates that decision-makers prefer to $f_{2}$.

The results are calculated by the methods used in the literature [21-23], that is $(160,195)$, which is completely consistent with the result of the first case of this example which illustrates the effectiveness of the improved S-type membership function method. In the multiobjective transportation decision-making problem, the decision-makers can select the optimal compromise solution according to their actual conditions and referring to Tables 4-6 of this paper.

\section{Conclusion}

For the multiobjective transportation decision-making problem, the decision-makers must determine the optimal compromise solution from a set of nondominated solutions. For the multiobjective transportation decision-making problem with larger $m$ and $n$, it is difficult to find the optimal compromise solution when the objective units are not consistent. However, the fuzzy compromise programming method with an improved S-type membership function is proposed in this paper. Meanwhile, by considering each objective and the overall objective, the fuzzy compromise programming method can find the optimal compromise solution. By adjusting the weight $w$ and the global evaluation parameter $\alpha$, the marginal evaluation of a single objective and the global evaluation of all objectives are comprehensively measured. However, in the actual engineering problem of multiobjective logistics transportation, the determination of the weight of the objective function is an important issue, which needs to be studied in detail based on actual problems. The S-type membership fuzzy compromise programming method proposed in this paper is more suitable for solving multitransportation problems and other multiobjective programming problems.

\section{Data Availability}

The data used to support the findings of this study are included in the paper.

\section{Conflicts of Interest}

The authors declare that there are no conflicts of interest regarding the publication of this paper.

\section{Acknowledgments}

This research was supported by the National Natural Science Foundation of China (51809203, 51709214, and 51779200) and supported by the Fundamental Research Funds for the Central Universities (215202003).

\section{References}

[1] H. J. Zimmermann, "Fuzzy programming and linear-programming with several objective functions," Fuzzy Sets and Systems, vol. 1, no. 1, pp. 46-55, 1978.

[2] J. Current and H. Min, "Multiobjective design of transportation networks: taxonomy and annotation," European Journal of Operational Research, vol. 26, no. 2, pp. 187-201, 1986.

[3] J. Current and M. Marsh, "Multiobjective transportation network design and routing problems: taxonomy and annotation," European Journal of Operational Research, vol. 65, no. 1, pp. 4-19, 1993.

[4] J. N. Clímaco, C. Henggeler Antunes, and M. J. Alves, "Interactive decision support for multiobjective transportation problems," European Journal of Operational Research, vol. 65, no. 1, pp. 58-67, 1993.

[5] J. L. Ringuest and D. B. Rinks, "Interactive solutions for the linear multiobjective transportation problem," European Journal of Operational Research, vol. 32, no. 1, pp. 96-106, 1987.

[6] O. İlker Kolak, O. Feyzioğlu, and N. Noyan, "Bi-level multiobjective traffic network optimization with sustainability perspective," Expert Systems with Applications, vol. 104, no. 4, pp. 294-306, 2018.

[7] C. Bai, B. Fahimnia, and J. Sarkis, "Sustainable transport fleet appraisal using a hybrid multi-objective decision making approach," Annals of Operations Research, vol. 250, no. 2, pp. 309-340, 2017.

[8] Z. Luo, "Path selection of multimodal transport based on multi-objective mixed integer programming," Journal of Transportation Technologies, vol. 9, no. 4, pp. 462-473, 2019. 
[9] J.-Y. Dong and S.-P. Wan, "A new method for solving fuzzy multi-objective linear programming problems," Iranian Journal of Fuzzy Systems, vol. 16, no. 3, pp. 145-159, 2019.

[10] J.-Y. Dong and S.-P. Wan, "A new trapezoidal fuzzy linear programming method considering the acceptance degree of fuzzy constraints violated," Knowledge-Based Systems, vol. 148, pp. 100-114, 2018.

[11] S.-P. Wan and J.-Y. Dong, "Possibility linear programming with trapezoidal fuzzy numbers," Applied Mathematical Modelling, vol. 38, no. 5-6, pp. 1660-1672, 2014.

[12] J. Matejaš and T. Perić, "A new iterative method for solving multiobjective linear programming problem," Applied Mathematics and Computation, vol. 243, pp. 746-754, 2014.

[13] F. R. Lima-Junior and L. C. R. Carpinetti, "A multicriteria approach based on fuzzy QFD for choosing criteria for supplier selection," Computers \& Industrial Engineering, vol. 101, no. 11, pp. 269-285, 2016.

[14] J. Qin, X. Liu, and W. Pedrycz, "An extended TODIM multicriteria group decision making method for green supplier selection in interval type-2 fuzzy environment," European Journal of Operational Research, vol. 258, no. 2, pp. 626-638, 2017.

[15] H. Dalman, “A Fuzzy programming approach for interval multiobjective solid transportation problem," New Trends in Mathematical Science, vol. 4, no. 4, p. 114, 2016.

[16] S. Cheng, H. Zhan, and H Yao, "Large-scale many-objective particle swarm optimizer with fast convergence based on alpha-stable mutation and Logistic function," Applied Soft Computing, vol. 99, no. 3, Article ID 106947, 2020.

[17] Y. Li, J. Li, J. He, and S. Zhang, "The real-time pricing optimization model of smart grid based on the utility function of the logistic function," Energy, vol. 224, no. 11, Article ID 120172, 2021.

[18] Z. Xiang, J. Yang, M. H. Naseem, Z. Xiang, and X. Liang, "Supplier selection of shipbuilding enterprises based on intuitionistic fuzzy multicriteria decision," Mathematical Problems in Engineering, vol. 2021, Article ID 1775053, 11 pages, 2021.

[19] M. Dharmadas, M. Gurupada, and R. Sankar Kumar, "Solving bi-level multi-objective transportation problem under fuzziness," International Journal of Uncertainty, Fuzziness and Knowledge-Based Systems, vol. 29, no. 3, pp. 411-433, 2021.

[20] U. M. Sharif, M. Musa, M. A.-A. Khan, and A. L. A. Ali, "Goal programming tactic for uncertain multi-objective transportation problem using fuzzy linear membership function," Alexandria Engineering Journal, vol. 60, no. 2, pp. 2525-2533, 2021.

[21] M. Zangiabadi and H. R. Maleki, "Fuzzy goal programming for multiobjective transportation problems," Journal of Applied Mathematics \& Computing, vol. 24, no. 1-2, pp. 449-460, 2007.

[22] R. Verma, M. P. Biswal, and A. Biswas, "Fuzzy programming technique to solve multi-objective transportation problems with some non-linear membership functions," Fuzzy Sets and Systems, vol. 91, no. 1, pp. 37-43, 1997.

[23] A. K. Bit, M. P. Biswal, and S. S. Alam, "Fuzzy programming approach to multicriteria decision making transportation problem," Fuzzy Sets and Systems, vol. 50, no. 2, pp. 135-141, 1992. 